\title{
Optimization of Dental Devices and Tools Used on Teeth
}

\author{
Berna Uzun $\mathbb{D}^{1,2}$ Dilber Uzun Ozsahin, ${ }^{1,3,4}$ Hani Hamdan, ${ }^{5}$ Jinan Charafeddine, ${ }^{6}$ \\ Gürkan Ünsal, ${ }^{1,7}$ and Deniz Özyer $\mathbb{1}^{3}$ \\ ${ }^{1}$ Near East University, DESAM Research Institute, Nicosia, TRNC, Mersin 10, Turkey \\ ${ }^{2}$ Near East University, Department of Mathematics, Nicosia, TRNC, Mersin 10, Turkey \\ ${ }^{3}$ Near East University, Department of Biomedical Engineering, Nicosia, TRNC, Mersin 10, Turkey \\ ${ }^{4}$ University of Sharjah, College of Health Science, Medical Diagnostic Imaging Department, Sharjah, UAE \\ ${ }^{5}$ Université Paris-Saclay, CentraleSupélec, CNRS, Laboratoire des Signaux et Systèmes (L2S UMR CNRS 8506), Gif-sur-Yvette, France \\ ${ }^{6}$ Université Paris-Saclay, Laboratoire d'Ingénierie des Systèmes de Versailles (LISV EA4048), Vélizy, France \\ ${ }^{7}$ Near East University, Faculty of Dentistry, Department of Dentomaxillofacial Radiology, Nicosia, TRNC, Mersin 10, Turkey
}

Correspondence should be addressed to Berna Uzun; berna.uzun@neu.edu.tr

Received 23 March 2021; Revised 17 May 2021; Accepted 4 June 2021; Published 9 August 2021

Academic Editor: Li Wu Zheng

Copyright (c) 2021 Berna Uzun et al. This is an open access article distributed under the Creative Commons Attribution License, which permits unrestricted use, distribution, and reproduction in any medium, provided the original work is properly cited.

\begin{abstract}
Taking decisions is important in every aspect of life. Decision-making has become a difficult problem in any situation where there are multiple criteria. The application of multicriteria decision-making methods that can bring mathematical and logical solutions to the problem from an analytical perspective has experienced considerable growth recently. It provides great benefits in solution and subsequent stages. Medical equipment selection is also a challenging, complex, and difficult problem for the decision-maker, due to the requirements of conflicting criteria, which must be taken into account simultaneously. In this context, the aim of this study implicates the principle of multicriteria decision-making theories on various types of instruments used in dentistry. Since the data used in this study are not numeric but linguistic, the Fuzzy PROMETHEE decision-making method is used. In this research, six dental tools most commonly used by professionals to perform operations on patients are compared and evaluated. Fuzzy PROMETHEE decision-making method investigations show that the dental mirror is the most effective tool among all compared tools, followed by dental suction, dental air abrasion, dental handpiece, dental laser, and dental X-ray, consequently, basing on the selected criteria and the importance weight given to each criterion. Using this technique, one can obtain more specific ranking results based on a specific preference level.
\end{abstract}

\section{Introduction}

Dental health is a critical factor in determining one's quality of life. The teeth are the only bone tissue in the human body that is not covered by skin. Subsequently, they are the most sensitive bone tissue in the body. Dentists often face difficulties intervening in the disease of the oral cavity. The tools used are critical and important in diagnosing dental caries, periodontal diseases, oral cancers, oral manifestations of HIV, oro-dental trauma, cleft lip and palate, and noma (severe gangrenous disease starting in the mouth, mostly affecting children).

These devices may or may not be electronic. They enable a doctor or a dentist to detect health problems and determine what kind of treatment is to be indicated. However, there are a big variety of medical devices and tools, as well as many types of illnesses. Therefore, this study focuses on a few dental problems and then discusses six types of mostly used and easily accessible dental tools and devices,with their advantages and disadvantages. In this research, the focus is on the use of medical devices for dental pathologies. Also, this research covers those devices that can be used in hospitals and dental clinics.

The focus of this paper is to evaluate the benefits of dental tools/devices and how they make dental treatments easier and more efficient. This paper will explain certain treatments, followed by a discussion on some tools and devices used in dentistry. Finally, this study will provide insight into the 
future of dentistry equipment and how it can be used to provide services that will make patients happy when they leave the dentist's office. This equipment is composed of several components, some of which have removable heads; it is critical to understand how to replace those components. During the majority of procedures (i.e., operation), the majority of these devices, such as suction, must be used continuously.

The decision-making process is the ability of the decision-makers to choose the most suitable alternative for their purposes and according to determined criteria. Multicriteria decision-making (MCDM) is a subbranch of the decision-making process. There are different types of MCDM techniques available for different types of decision matrix, which contains the parameters of the alternatives, and it becomes more complicated when conflicting criteria occur. These techniques are not applicable where there is uncertain information arising in decision problems. In such cases, fuzzy modeling enables the decision-maker to define the problem mathematically and obtain a solution. However, the comparison of fuzzy numbers is a complex part of the fuzzy MCDM problems. Additionally, it contains the most important part of these problems. The selection problems mostly contain uncertain parameters; therefore, defining the problem by considering the uncertainties requires fuzzy modeling and comparison between the fuzzy sets. There are numerous types of fuzzy MCDM modeling methods available for the comparison of alternatives in a fuzzy environment [1-3]. Yager first presented the centroid method for comparing and ranking fuzzy numbers, even though this technique was known for a hundred years [4]. After Yager's study, different types of ranking indices of the fuzzy sets have been proposed by numerous studies [5-7]. Dong and Wan proposed a new method of PROMETHEE-fuzzy linear programming to give a rational solution to multiattribute group decision-making problems where the decision matrix contains heterogeneous information such as real numbers, intuitionistic fuzzy sets, intervals, triangular intuitionistic fuzzy numbers, and trapezoidal fuzzy numbers, and where the importance weight is not precise [8]. Wan et al. defined the left-right geometric consistency of the triangular multiplicative preference relation by including the experts' trust level in the model and provided a new decision-making method for individuals and for the group in order to extract the experts' weights [9]. Dong et al. proposed the best-worst method for the optimistic and pessimistic decision-maker to define the fuzzy weight vector for the multicriteria decision problems with the fuzzy consistency index [10]. Wan and Dong gave detailed information on interval-valued intuitionistic fuzzy sets and their application in decision-making theories [11]. Wan et al. provided the left-right geometric consistency definition of the triangular multiplicative preference relations (TRMF) by considering the trust levels of the experts in order to create a model with an acceptable consistent TMPR, and that study has proposed two algorithms for the individual and group decision-making problems [12]. Those studies are also beneficial for the comparison of fuzzy numbers. However, the strength of the Yager index for the optimization of fuzzy sets is also shown [13].
In this paper, the Fuzzy PROMETHEE technique, which uses multicriteria decision-making for choosing correct dental device characteristics, is implemented.

1.1. Dental Mirror. A dental mirror is a probe with a thin circular mirrored surface on the working end that allows the dentist to see the inside of the teeth and the rest of the oral cavity. This instrument is used to search for bacteria, cavities, and calculus in hard-to-see areas of the mouth. A dental mirror costs about \$10. Dentists use three different types of mirrors: front surface, concave, and plane surface. Since it offers a clear image, the front surface mirror is the most frequently used dental mirror [14].

1.2. Dental Probe (Dental Handpiece). A dental probe is a pointed instrument used by dentists to diagnose dental problems rather than treat them. A dental probe is a hand-held instrument with a curved end and a blunt, pointed tip. It is used to diagnose and assess dental diseases and conditions. There are many styles of explorers, including straight, interproximal, and curved. A dental probe is around $\$ 200$ [15]. Dental probes are used to determine the depth of periodontal pockets formed by teeth. Periodontal depth is critical since abscessed teeth are often associated with advanced periodontal disease, necessitating gum surgery. Therefore, it is important to check the margin regularly to identify any issues before they become significant and the tooth cannot be saved [16].

1.3. Dental Laser. Although they are relatively new, dental lasers are already being used to treat a range of conditions, including soft and hard tissue, gums and teeth, caries detection, biostimulation, low-level laser therapy, and photostimulation. Dentists use this device to remove diseased dental tissue and prepare the tooth for dental reconstruction. Dental lasers are used by some dentists to treat conditions such as tooth decay, gum disease, biopsy, and teeth whitening. As is the case for many devices, this one has several advantages and disadvantages. However, since the dental laser is a relatively new method, it has created controversy in the dental community. Several benefits include decreased physical discomfort and anxiety for the patient. When the laser was used instead of more conventional methods, less bleeding was detected during operations. Some drawbacks include the fact that it is more expensive than other tools. It is over $\$ 1000$ in price [17].

1.4. Dental Suction. Dental suctions, also known as saliva ejectors, are used by dentists to remove excess saliva from patients' mouths during care. Additionally, dentists use the system to remove any unnecessary dental materials. Teeth must remain clean, dry, and free of blood, saliva, and water. If the patient has saliva or dental materials in his or her mouth, the operation may be delayed. Because it is a dental appliance, it is difficult to clean after each patient. Rather than that, it is simpler to replace it with a sterile tip for the next patient. These tips are frequently made of plastic or metal. The tips can be disposable or autoclavable, which requires 15 to 20 minutes to complete the sterilization cycle. Since certain procedures can cause the patient to gag, which 
may result in errors, dentists may use this device for the patient's comfort. Dental suction costs about \$600 [18].

1.5. Dental Air Abrasion. Dental air abrasion is one of the most costly pieces of equipment reviewed in this study. It costs approximately $\$ 700$, while the dental mirror costs about $\$ 10$. While they are similar in scale, their functions and costs are very different. The dental mirror is used to locate the problem, and dental air abrasion is used to remove decay from the tooth by blowing a stream of aluminum oxide-containing air onto the tooth [19].

1.6. Dental X-Ray. X-ray is often used to provide a better view of the teeth and their roots. Dentists may use dental X-rays to detect cavities, their depth, and the presence of any other conditions before initiating treatment. Additionally, it shows the emerging teeth that are still under the gums. Dental Xrays can diagnose oral health issues in patients early on, including oral infections, some types of tumors, and gum disease. The dental X-ray is the most expensive instrument discussed in this article. It is both because it is a large electronic system and because it is essential in dentistry. Dental X-rays have evolved significantly since the early years of the twentyfirst century. As a result, it is unsurprising that dental X-rays cost about $\$ 800[20]$.

\section{Material and Methodology}

2.1. PROMETHEE (Preference Ranking Organization Method for Enrichment Evaluation). The PROMETHEE (Preference Ranking Organization Method for Enrichment Evaluation) method technique developed by Professor Jean-Pierre Brans in 1982 [21, 22] is one of the easiest and most efficient models compared to other multicriteria decision-making methods. The best solution to the problem is obtained by comparing each criterion selected for each device. This method is a fair comparison of variants from the point of view of all criteria step by step. Only two types of parameters are required in the PROMETHEE model: each separate criterion and weights of the criteria.

In this context, using this MCDA (multicriteria decision analysis) technique gained so many advantages. A userfriendly outranking method is easy and has been successfully applied to real-life planning problems. PROMETHEE I and PROMETHEE II give a partial and net ranking of the alternatives, respectively, while still satisfying simplicity [23]. follows.

The main process of the PROMETHEE technique is as

After the collection of the decision matrix, each selected criterion $j$, the preference function $p_{j}(d)$, and the important degrees of each criterion $\left(w_{k}\right)$ will be determined by the decision-makers. Then, the outranking relation $\left(\pi\left(a_{t}, a_{t^{\prime}}\right)\right)$ should be calculated for each pair of alternatives $\left(a_{t}\right.$ and $a_{t^{\prime}}$ $\in A$ ) using

$$
\pi\left(a_{t}, a_{t^{\prime}}\right)=\sum_{k=1}^{K} w_{k} \cdot\left[p_{k}\left(f_{k}\left(a_{t}\right)-f_{k}\left(a_{t^{\prime}}\right)\right)\right], A X A \longrightarrow[0,1] .
$$

$\pi(a, b)$ denotes the preference index where $k$ denotes the $k$-th criteria. This index shows the preference intensity of $a_{t}$ compared to $a_{t^{\prime}}$ by considering each criterion simultaneously.

Then, the positive outranking flow $\left(\Phi^{+}\left(a_{t}\right)\right)$ and negative outranking flow $\left(\Phi^{-}\left(a_{t}\right)\right)$ should be counted by using Equations (2) and (3) sequentially.

(i) The positive outranking flow of alternative $a_{t}$ :

$$
\Phi^{+}\left(a_{t}\right)=\frac{1}{n-1} \sum_{\substack{t^{\prime}=1 \\ t^{\prime} \neq t}}^{n} \pi\left(a_{t}, a_{t^{\prime}}\right) .
$$

(ii) The negative outranking flow of alternative $a_{t}$ :

$$
\Phi^{-}\left(a_{t}\right)=\frac{1}{n-1} \sum_{\substack{t^{\prime}=1 \\ t^{\prime} \neq t}}^{n} \pi\left(a_{t^{\prime}}, a_{t}\right)
$$

where $n$ denotes the number of alternatives.

The positive outranking flow is the degree of dominating other alternatives while the negative outranking flow is the degree of being dominated by the other alternatives. Therefore, based on the positive and negative outranking flows, the partial preorder of the alternatives can be obtained based on the following cases:

Case 1. Alternative $a_{t}$ should be preferred to the alternative $a_{t^{\prime}}\left(a_{t} P a_{t^{\prime}}\right)$ if

$$
\left\{\begin{array}{l}
\Phi^{+}\left(a_{t}\right)>\Phi^{+}\left(a_{t^{\prime}}\right), \Phi^{-}\left(a_{t}\right) \leq \Phi^{-}\left(a_{t^{\prime}}\right) \\
\Phi^{+}\left(a_{t}\right)=\Phi^{+}\left(a_{t^{\prime}}\right), \Phi^{-}\left(a_{t}\right)<\Phi^{-}\left(a_{t^{\prime}}\right)
\end{array}\right.
$$

Case 2. $a_{t}$ is not different from $a_{t^{\prime}}\left(a_{t} I a_{t^{\prime}}\right)$ if

$$
\left(a_{t} I a_{t^{\prime}}\right) \text { if : } \Phi^{+}\left(a_{t}\right)=\Phi^{+}\left(a_{t^{\prime}}\right), \Phi^{-}\left(a_{t}\right)=\Phi^{-}\left(a_{t^{\prime}}\right)
$$

Case 3. $a_{t}$ is incomparable to $a_{t^{\prime}}\left(a_{t} R a_{t^{\prime}}\right)$ if

$$
\left\{\begin{array}{l}
\Phi^{+}\left(a_{t}\right)>\Phi^{+}\left(a_{t^{\prime}}\right), \Phi^{-}\left(a_{t}\right)>\Phi^{-}\left(a_{t^{\prime}}\right) \\
\Phi^{+}\left(a_{t}\right)<\Phi^{+}\left(a_{t^{\prime}}\right), \Phi^{-}\left(a_{t}\right)<\Phi^{-}\left(a_{t^{\prime}}\right) .
\end{array}\right.
$$

If the $3^{\text {rd }}$ case occurs during partial preorder calculation, applyed to the PROMETHEE II, the net outranking values $\left(\Phi^{\text {net }}\left(a_{t}\right)\right)$ should be calculated using

$$
\Phi^{\mathrm{net}}\left(a_{t}\right)=\Phi^{+}\left(a_{t}\right)-\Phi^{-}\left(a_{t}\right)
$$

And the net ranking results of the alternatives should be determined based on the following cases: 
Case 1. $a_{t}$ is preferred to

$$
a_{t^{\prime}}\left(a_{t} P a_{t^{\prime}}\right) \text { if } \Phi^{\text {net }}\left(a_{t}\right)>\Phi^{\text {net }}\left(a_{t^{\prime}}\right)
$$

Case 2. $a_{t}$ is not different than

$$
a_{t^{\prime}}\left(a_{t} I a_{t^{\prime}}\right) \text { if } \Phi^{\text {net }}\left(a_{t}\right)=\Phi^{\text {net }}\left(a_{t^{\prime}}\right)
$$

The most preferable alternative should have a higher net outranking flow.

2.2. Fuzzy PROMETHEE (F-PROMETHEE). Fuzzy logic is a multivalued logic that describes vague conditions more rationally $[24,25]$. Real-life problems often involve vague situations that are often difficult to quantify numerically and can only be expressed linguistically. The Fuzzy PROMETHEE (F-PROMETHEE) method has been developed as a hybrid model because of the insufficient PROMETHEE method in such problems. The main aim of the Fuzzy PROMETHEE model is to propose a comparison between two fuzzy sets. There have been few types of research that applied Fuzzy PROMETHEE to make an optimal decision on different realistic problems such as cancer treatment techniques, nuclear medicine and oncology, analysis of image reconstruction, and X-ray-based medical imaging devices [23, 26-31]. Therefore, in this study, the F-PROMETHEE method is used to ensure that the input data are interpreted correctly.

\section{Results}

During the first evaluation, the six most frequently used dental devices (such as dental mirror, dental laser, dental suction, dental air abrasion, dental X-ray, and dental probe) were evaluated and compared using the Fuzzy PROMETHEE decision-making method. These criteria include cost, calibration, period, practicality, advantages, disadvantages, comfortability, dose, and size, as shown in Table 1.

The simulation of devices carried out shows the advantages and disadvantages of these devices according to different criteria. The result shows that the devices have different points on the scale. These devices have different positive and negative values. For example, the cost of a dental mirror is approximately $\$ 10$, while a dental probe is around $\$ 200$. While they have different purposes, a dental mirror is cheaper. The dental laser is used for treatments, while the dental mirror is used for diagnosis. Also, the dental mirror does not have anything mechanical or electrical while the dental laser does. Thus, it makes sense for the dental laser to cost so much compared to the dental mirror. The dental mirror is used to identify medical problems, while a dental air abrasion is used to remove decay from the tooth by blowing a stream of aluminum oxide as air into the tooth. While the dental mirror gives immediate results and is temporary, the dental X-ray gives a more permanent image that can be observed for a longer period without making the patient tired. While the dental probe costs approximately $\$ 200$, dental air abrasion costs about $\$ 700$. Both of these have a curvy end; However, the dental air abrasion has an end that has a tiny hole, enabling it to spray particles onto the tooth. Instead of that, the dental probe has a sharp end.

Since the data collected for the analysis of dental devices are not numerical, the linguistic fuzzy scale has been used to convert the obtained dataset to numerical values. As a result, the linguistic fuzzy scale is used as shown in Table 2.

The Yager Index (YI $=3 n-a+(b / 3))$ of the fuzzy number $\tilde{F}=(n-a, n, n+b)$ is applied to defuzzify the linguistic data.

In this study, the input data are also treated as fuzzy numbers. This took into account the uncertainty contained in the data, which could give more valuable ranking results by considering the fuzziness. After the defuzzification, the PROMETHEE process is then employed for the ranking of dental devices with minimizing cost, disadvantages, dose, and size of the alternatives and maximizing the advantages and comfortability. In this analysis, the calibration period and practicability are not used as criteria since there is no difference between the alternatives corresponding to these criteria. Also, the importance weight of the criteria has been chosen equally.

According to the ranking result, the dental mirror is the most effective tool among others as shown in Table 3. These results were obtained on the basis of the criteria. The complete ranking is obtained according to the selected weights. According to Table 3, the best solutions are those with the highest net flow. Table 3 shows the complete ranking of dental devices according to the selected criterion, which is necessary for the performance of the devices. At least, these simulations of filling devices are important and effective for the dental industry.

To test the results obtained with Fuzzy PROMETHEE for the evaluation of the dental devices, the fuzzy TOPSIS (Technique for Order of Preference by Similarity to Ideal Solution) technique is used, which is also an another successfully used MCDA technique. The TOPSIS technique has been defined by Hwang and Yoon in 1981 [30]. It evaluates the alternatives based on their distance to the positive ideal solution $\left(A^{+}\right)$and the negative ideal solution $\left(A^{-}\right)$under conflicting criteria. The positive ideal solution is the combination of the best values of each criterion, while the negative ideal solution is the combination of the worst values of each criterion. It applies to the selection problems with the numerical dataset. Furthermore, relative closeness to the positive ideal solution $\left(R_{i}\right)$ should be considered as the most preferred alternative with this technique. This is a ratio that can be counted based on the distance of alternatives to the positive ideal solution $\left(d^{+}\right)$and distance of the alternatives to negative ideal solution $\left(d^{-}\right)$based on

$$
R_{i}=\frac{d_{i}^{-}}{d_{i}^{-}+d_{i}^{+}}
$$

After demulsifying the data of the dental devices and normalizing the decision matrix, the weighted normalized matrix is computed, and then the positive and the negative ideal solutions are obtained as shown in Table 4. 
TABLe 1: Simulation of dental devices.

\begin{tabular}{lcccccccccccc}
\hline & Dental device & Cost $(\$)$ & Calibration period & Practicality & Advantages & Disadvantages & Comfortability & Dose & Size \\
\hline 1 & Dental mirror & 10 & Daily & Yes & Medium & Low & Comfortable & None & Low \\
2 & Dental handpiece & 200 & Daily & Yes & Medium & Medium & Comfortable & Low & Low \\
3 & Dental laser & 500 & Daily & Yes & High & Medium & Comfortable & Medium Medium \\
4 & Dental suction & 600 & Daily & Yes & High & Very low & Comfortable & None & Low \\
5 & Dental air abrasion & 700 & Daily & Yes & Medium & Low & Comfortable & Very low & Low \\
6 & Dental X-ray & 800 & Daily & Yes & Very high & High & Comfortable & Low & High \\
\hline
\end{tabular}

TABLE 2: Selected fuzzy scale for the linguistic data.

\begin{tabular}{lc}
\hline Linguistic scale for evaluation & Triangular fuzzy scale \\
\hline Very high (VH) & $(0.75,1,1)$ \\
Important $(\mathrm{H})$ & $(0.50,0.75,1)$ \\
Medium (M) & $(0.25,0.50,0.75)$ \\
Low (L) & $(0,0.25,0.50)$ \\
Very low (VL) & $(0,0,0.25)$ \\
\hline
\end{tabular}

TAble 3: Fuzzy PROMETHEE ranking results.

\begin{tabular}{lcccc}
\hline \multirow{2}{*}{ Complete ranking } & Dental device & $\Phi$ & $\Phi^{+}$ & $\Phi^{-}$ \\
\hline 1 & Dental mirror & 0.0076 & 0.0079 & 0.0003 \\
2 & Dental suction & 0.0056 & 0.0063 & 0.0007 \\
3 & Dental air abrasion & 0.0039 & 0.0054 & 0.0016 \\
4 & Dental handpiece & -0.0003 & 0.0034 & 0.0037 \\
5 & Dental laser & -0.0056 & 0.0007 & 0.0063 \\
6 & Dental X-ray & -0.0111 & 0.0007 & 0.0118 \\
\hline
\end{tabular}

Table 4: Positive and negative ideal solutions of dental devices.

\begin{tabular}{lcc}
\hline Criteria & Aim $\min / \max$ & $A^{+} / A^{-}$ \\
\hline Cost $(\$)$ & Min & $0.0078 / 0.0902$ \\
Advantages & Max & $0.0929 / 0.0505$ \\
Disadvantages & Min & $0.0122 / 0.1144$ \\
Comfortability & Max & $0.0962 / 0.0000$ \\
Dose & Min & $0.0000 / 0.1349$ \\
Size & Min & $0.0404 / 0.1213$ \\
\hline
\end{tabular}

TABLE 5: Fuzzy TOPSIS ranking results.

\begin{tabular}{lccc}
\hline Ranking & Alternatives & $d^{+} / d^{-}$ & $R_{i}$ \\
\hline 1 & Dental mirror & $0.0497 / 0.2159$ & 0.8128 \\
2 & Dental suction & $0.0679 / 0.2130$ & 0.7582 \\
3 & Dental air abrasion & $0.0986 / 0.1856$ & 0.6530 \\
4 & Dental handpiece & $0.1414 / 0.1299$ & 0.4787 \\
5 & Dental X-ray & $0.1939 / 0.0797$ & 0.2914 \\
6 & Dental laser & $0.1945 / 0.0633$ & 0.2455 \\
\hline
\end{tabular}

\section{Data Availability}

The fuzzy data used to support the findings of this study are available from the corresponding author upon request. 


\section{Conflicts of Interest}

The authors declare no conflict of interest.

\section{References}

[1] R. Yager, "A procedure for ordering fuzzy subsets of the unit interval," Information Sciences, vol. 24, no. 2, pp. 143-161, 1981.

[2] X. Wang and E. E. Kerre, "Reasonable properties for the ordering of fuzzy quantities (I)," Fuzzy Sets and Systems, vol. 118, no. 3, pp. 375-385, 2001.

[3] K.-P. Chiao, "A new ranking approach for general interval type-2 fuzzy sets using extended alpha cuts representation," in 2015 10th International Conference on Intelligent Systems and Knowledge Engineering (ISKE), pp. 594-597, Taipei, Taiwan, 2015.

[4] R. R. Yager, "On a general class of fuzzy connectives," Fuzzy Sets and Systems, vol. 4, no. 3, pp. 235-242, 1980.

[5] S. J. Chen and S. M. Chen, "Fuzzy risk analysis based on the ranking of generalized trapezoidal fuzzy numbers," Applied Intelligence, vol. 26, no. 1, pp. 1-11, 2007.

[6] C. Liang, J. Wu, and J. Zhang, "Ranking Indices and Rules for Fuzzy Numbers based on Gravity Center Point," in 2006 6th World Congress on Intelligent Control and Automation, pp. 3159-3163, Dalian, China, 2006.

[7] Y. J. Wang and H. S. Lee, "The revised method of ranking fuzzy numbers with an area between the centroid and original points," Computers and Mathematics with Applications, vol. 55, no. 9, pp. 2033-2042, 2008.

[8] J. Dong and S. Wan, "A PROMETHEE-FLP method for heterogeneous multi-attributes group decision making," IEEE Access, vol. 6, pp. 46656-46667, 2018.

[9] S. Wan, W. Zou, L. Zhong, and J. Dong, "Some new information measures for hesitant fuzzy PROMETHEE method and application to green supplier selection," Soft Computing, vol. 24, no. 12, article 4446, pp. 9179-9203, 2020.

[10] J. Dong, S. Wan, and S. Chen, "Fuzzy best-worst method based on triangular fuzzy numbers for multi-criteria decision-making," Information Sciences, vol. 547, pp. 1080-1104, 2021.

[11] S. P. Wan and J. Y. Dong, Decision Making Theories and Methods Based on Interval-Valued Intuitionistic Fuzzy Sets, Springer, Singapore, 1st ed edition, 2020.

[12] S. Wan, X. Cheng, C. Chen, and J. Dong, "L-R geometric consistency definition of triangular multiplicative preference relation in group decision making," Fuzzy Sets and Systems, vol. 409, pp. 85-113, 2021.

[13] J. Figueroa-Garcia, Y. Chalco-Cano, and H. Roman-Flores, "Yager index and ranking for interval type-2 fuzzy numbers," IEEE Transactions on Fuzzy Systems, vol. 26, no. 5, pp. 27092718, 2018.

[14] M. Castro, A. Videira, Z. Luciane, K. Carlos, P. Carlos, and A. Bovi, "Comparison of diagnostic methods for dental caries," Journal of dentistry for children, vol. 70, no. 2, pp. 115-119, 2003.

[15] 2019, https://www.altex.com/GC-5-12-Curved-Dental-Pick12-2266-P143468C10778.aspx/.

[16] “The detailed clinical periodontal examination," 2019, https:// pocketdentistry.com/8-the-detailed-clinical-periodontalexamination/.
[17] R. Convissar, Principles and Practice of Laser Dentistry, vol. 2015, Elsevier, New York, 2nd Edition edition, 2015.

[18] "Mouth mirror that works as suction also," 2019, https://www. indiegogo.com/projects/dental-mouth-mirror-with-suction/.

[19] V. S. Hegde and R. Khatavkar, "A new dimension to conservative dentistry: air abrasion," Journal of Conservative Dentistry, vol. 13, no. 1, article 62632, pp. 4-8, 2010.

[20] A. Gulson and J. Holroyd, "Guidance on the safe use of handheld dental X-ray equipment," in Chemical and Environmental Hazards Public Health England, Chilton Information Office Centre for Radiation, England, 2016.

[21] J. Brans and P. Vincke, "Note-A Preference Ranking Organisation Method," Management Science, vol. 31, no. 6, pp. 647656, 1985.

[22] J. Brans, P. Vincke, and B. Mareschal, "How to select and how to rank projects: the PROMETHEE method," European Journal of Operational Research, vol. 24, no. 2, pp. 228-238, 1986.

[23] D. Uzun, B. Uzun, M. Sani et al., "Evaluating cancer treatment Alternatives using fuzzy PROMETHEE method," International Journal of Advanced Computer Science and Applications, vol. 8, no. 10, pp. 177-185, 2017.

[24] B. Uzun and E. Kiral, "Forecasting closing returns of Borsa Istanbul index with Markov chain process of fuzzy states," Pressacademia, vol. 4, no. 1, pp. 15-24, 2017.

[25] B. Uzun and E. Kiral, "Application of Markov chains-fuzzy states to gold price," Procedia Computer Science, vol. 120, pp. 365-371, 2017.

[26] D. U. Ozsahin, B. Uzun, M. Musa, N. Şentürk, F. Nurçin, and I. Ozsahin, "Evaluating nuclear medicine imaging devices using fuzzy PROMETHEE method," Procedia Computer Science, vol. 120, pp. 699-705, 2017.

[27] D. Uzun, B. Uzun, M. Sani, and I. Ozsahin, "Evaluating X-ray based medical imaging devices with fuzzy preference ranking organization method for enrichment evaluations," International Journal of Advanced Computer Science and Applications, vol. 9, no. 3, pp. 7-10, 2018.

[28] I. Ozsahin, T. Sharif, D. Ozsahin, and B. Uzun, "Evaluation of solid-state detectors in medical imaging with fuzzy PROMETHEE," Journal of Instrumentation, vol. 14, no. 1, article C01019, 2019.

[29] D. U. Ozsahin and I. Ozsahin, “A fuzzy PROMETHEE approach for breast cancer treatment techniques," International Journal of Medical Research \& Health Sciences, vol. 7, no. 5, pp. 29-32, 2018.

[30] D. Uzun Ozsahin, N. Isa, B. Uzun, and I. Ozsahin, "Effective analysis of image reconstruction algorithms in nuclear medicine using fuzzy PROMETHEE," in 2018 Advances in Science and Engineering Technology International Conferences (ASET), pp. 1-5, Dubai, UAE, 2018.

[31] B. Uzun, M. Taiwo, A. Syidanova, and D. Uzun Ozsahin, “The Technique For Order of Preference by Similarity to Ideal Solution (TOPSIS)," in Application of Multi-Criteria Decision Analysis in Environmental and Civil Engineering, O. D. Uzun, H. Gökçekuş, B. Uzun, and J. LaMoreaux, Eds., pp. 25-30, Springer International Publishing, Cham, 2021. 